\title{
KRAS Gene Mutation in Patients with Primary Colorectal Cancer
}

Vu Thi Minh Thuc ${ }^{1}$, [MD] [PhD]

ORCID: 0000-0003-5752-6869

Le Van Thieu ${ }^{2}$, [MD] [PhD]

ORCID: 0000-0002-5752-6869

Huynh Quang Huy ${ }^{3}$, [MD] [PhD]

ORCID: 0000-0001-5177-1460

Nguyen Minh Duc ${ }^{3},[\mathrm{MD}]$

ORCID: 0000-0001-5411-1492

1 National Otorhinolaryngology Hospital of VietnamNational Otorhinolaryngology Hospital of Vietnam

2 Endoscopy department, Viet Tiep hospital, Vietnam

3 Radiology Department, Pham Ngoc Thach University of Medicine, Vietnam

* Corresponding Author: Huynh QuanHuy Address: Radiology Department, Pham Ngoc

Thach University of Medicine. 2 Duong Quang

Trung, District 10, Ho Chi Minh city, Vietnam

Phone numbers: +84982108108

e-mail : huyhq@pnt.edu.vn

DOI:10.32552/2019.ActaMedica.337

Received: 16 January 2018, Accepted: 26 February 2019

Published on line: 30 March 2019

\section{wee) ABSTRACT Cen}

Objective: KRAS mutation occurs in $30 \%$ to $50 \%$ of colorectal cancers. The aim of our study was to determine the frequency of KRAS mutations among patients with colorectal cancer; and the relationship with clinicopathologic features.

Materials and Methods: 79 colorectal cancer cases at a hospital in Hai Phong of Vietnam were collected, including 45 colon cancer and 34 rectal cancer during January 2010 and July 2012. PCR amplification and DNA sequencing were used to detect mutations in exon 2 of KRAS gene. The study was based on informed consent and approval by the Ethics Committee of Viet Tiep Hospital.

Results: KRAS mutation was found in $40.4 \%$ (225/557) colorectal cancer. All mutation locations were in codon 12 . There was significant association $(p<0.05)$ between KRAS mutations and tumor size, tumor stage or metastatic stage. No significant association was observed between KRAS mutations and gender, tumor location, tumor grade or histologic presence of mucin $(p>0.05)$.

Conclusion: Determining the KRAS mutational status of tumor samples has become an essential tool for managing patients with colorectal cancers.

Keywords: Colorectal cancer, KRAS gene mutation, clinicopathology.

\section{INTRODUCTION}

Colorectal cancer (CC) is one of the most prevalent diseases in the world and the third leading cause of death among cancer types. According to the Union for International Cancer Control (UICC), there are approximately 1 million new cases of $\mathrm{CC}$ each year in the world and more than 500,000 people die from it each year [1]. Incidence rates across various regions and continents are different. In Vietnam, CC is ranked fifth after stomach, lung, breast and nasopharynx cancers for mortality rates. Among gastrointestinal (Gl) cancers, CC is ranked as the second most prevalent cancer, after stomach cancer [2]. The mechanisms of initiation, development and progression of CC are attributed to gene modifications and accumulation of mutated genes in the mucosal cells of the colon. The gradual accumulation of genetic modifications often persists for many years (from 10 to 20 years), and this concurs with genetic and epidemiological studies that tumorigenesis progresses through many stages during that time [3].

With the rapid development of the biomedical industry, it is now possible to accurately, thoroughly and quickly identify almost all major types of mutations in the most common tumor cells, such as in lung cancer, malignant lymphomas, and colorectal cancer. Some studies on gene-modified mouse models have confirmed the importance of targeting genes in many various cancers, including KRAS oncogene in colorectal cancer [4]. From the above strategies, a new generation of anti-cancer drugs that accurately targets colon cancer cells can be 
developed- in a platform known as targeted therapy. In Vietnam, there have been some studies on CC, but almost all of them have focused on clinical characteristics, endoscopic imaging, and histopathology. There have only been a few studies investigating mutations in cancer cells, in general, and in CC, in particular.

In this present study, we aim to determine the frequency of KRAS mutations among patients with CC; and the relationship with clinicopathologic features.

\section{MATERIALS and METHODS}

\section{Patients}

We enrolled 79 CC patients at Viet Tiep Hospital of Hai Phong city. All patients were histologically diagnosed with CC during January 2010 and July 2012. Clinical data including patient's age and sex at diagnosis and primary tumor location were retrieved from patient records. Histopathological criteria, such as TNM classification, grade of differentiation (histological grade according to the WHO 2000 classification). We explored KRAS gene mutation by DNA extraction technique and gene sequencing technique at Gene and Protein Research Center of Ha Noi Medical University. The study was based on informed consent and approval by the Ethics Committee of Viet Tiep Hospital.

\section{DNA Extraction}

DNA was extracted from the patient's paraffin sample following the phenol/chloroform procedure after the precise selection of the cancerous tissue. Before extraction with phenol/chloroform, the paraffin was removed by xylene treatment.
DNA concentration and purity were measured on a Nano-Drop machine, and the DNA sample concentrations were quantified at OD 280/ OD260 (samples with purity $\geq 1.8$ were used for further experiments).

\section{Gene Sequencing}

After gene amplification of the codon region 12, 13 of the KRAS gene using nested Polymerase Chain Reaction (PCR), the PCR products were purified from the agarose gel using Promega Wizard SV gel clean-up system (Promega, Madison, WI, USA). The purified PCR products were sequenced using the BigDye terminator sequencing method (Applied Biosystems, Foster City, CA, USA). Gene sequences were compared to wild-type KRAS gene sequences on GeneBank (National Center for Biotechnology Information - NCBI).

\section{RESULTS}

Primary samples from 79 colorectal carcinoma patients were analyzed for KRAS gene mutations. Mutations of KRAS occurred in $58.2 \%$ (46/79) of CC. Mutated carcinomas were compared with non mutated carcinomas for sex, age, histological features and molecular characteristics (Table 1). Between subjects with KRAS mutation and KRAS wild type, there was no significant difference in age, sex, tumor location, histologic differentiation, and histologic presence of mucin. An association between KRAS mutation and tumor size $(p=0.0072)$, tumor stage (T stage) $(p=0.0179)$ or metastatic stage ( $M$ stage) $(p=0.0442)$ was observed significantly. Figure 1 show the gene sequencing of a male 71-year-old patient with the mutation at codon 12 .

Table 1. Relationship of KRAS status with clinicopathological features

\begin{tabular}{|ll|c|c|c|}
\hline Characteristics & $\begin{array}{c}\text { MutantKRAS } \\
(\mathrm{n}=46)\end{array}$ & $\begin{array}{c}\text { Wild-typeKRAS } \\
(\mathrm{n}=33)\end{array}$ & P-value \\
\hline Sex & Male & $29(60.4 \%)$ & $19(39.6 \%)$ & 0.6238 \\
& Female & $17(54.8 \%)$ & $14(45.2 \%)$ & 0.3293 \\
\hline Age & $\begin{array}{c}4(80.0 \%) \\
\text { s }\end{array}$ & $42(56.8 \%)$ & $32(43.2 \%)$ & $1(20.0 \%)$ \\
\hline
\end{tabular}


KRAS gene mutation in primary colorectal cancer patients.

\begin{tabular}{|c|c|c|c|}
\hline $\begin{array}{l}\text { Tumor location } \\
\text { Proximal colon } \\
\text { Distant colon } \\
\text { Rectal }\end{array}$ & $\begin{array}{l}12(70.6 \%) \\
13(46.4 \%) \\
21(63.6 \%)\end{array}$ & $\begin{array}{l}5(29.4 \%) \\
15(53.6 \%) \\
13(39.4 \%)\end{array}$ & 0.2411 \\
\hline $\begin{array}{l}\text { Tumor size } \\
\qquad \begin{array}{l}<5 \mathrm{~cm} \\
5-10 \mathrm{~cm} \\
>10 \mathrm{~cm}\end{array}\end{array}$ & $\begin{array}{c}2(4.3 \%) \\
15(32.6 \%) \\
29(63.1 \%)\end{array}$ & $\begin{array}{l}8(24.2 \%) \\
14(42.4 \%) \\
11(33.4 \%)\end{array}$ & 0.0072 \\
\hline $\begin{array}{l}\text { Differentiation degree (Grade) } \\
\text { Well (G1) } \\
\text { Moderate (G2) } \\
\text { Poor (G3) }\end{array}$ & $\begin{array}{l}19(59.4 \%) \\
15(55.6 \%) \\
12(60.0 \%)\end{array}$ & $\begin{array}{l}13(40.6 \%) \\
12(44.4 \%) \\
8(40.5 \%)\end{array}$ & 0.9406 \\
\hline $\begin{array}{l}\text { Presence of mucin } \\
\text { Mucinous } \\
\text { Non-mucinous }\end{array}$ & $\begin{array}{c}4(40.0 \%) \\
42(60.7 \%)\end{array}$ & $\begin{array}{c}6(60.0 \%) \\
27(39.3 \%)\end{array}$ & 0.2201 \\
\hline $\begin{array}{l}\text { pT stage } \\
\text { pT1-2 } \\
\text { pT3-4 }\end{array}$ & $\begin{array}{c}7(35.0 \%) \\
39(66.1 \%)\end{array}$ & $\begin{array}{l}13(65.0 \%) \\
20(33.9 \%)\end{array}$ & 0.0179 \\
\hline $\begin{array}{l}\text { pN stage } \\
\text { pN0 } \\
\text { pN1-2 }\end{array}$ & $\begin{array}{c}37(60.6 \%) \\
9(50.0 \%)\end{array}$ & $\begin{array}{l}24(39.4 \%) \\
9(50.0 \%)\end{array}$ & 0.4222 \\
\hline $\begin{array}{r}\text { pM stage } \\
\text { pM0 } \\
\text { pM1 }\end{array}$ & $\begin{array}{l}16(45,7 \%) \\
30(68,2 \%)\end{array}$ & $\begin{array}{l}19(54,3 \%) \\
14(31,8 \%)\end{array}$ & 0.0442 \\
\hline
\end{tabular}

Figure 1. Gene sequencing of a male 71-year-old patient with the mutation at codon 12
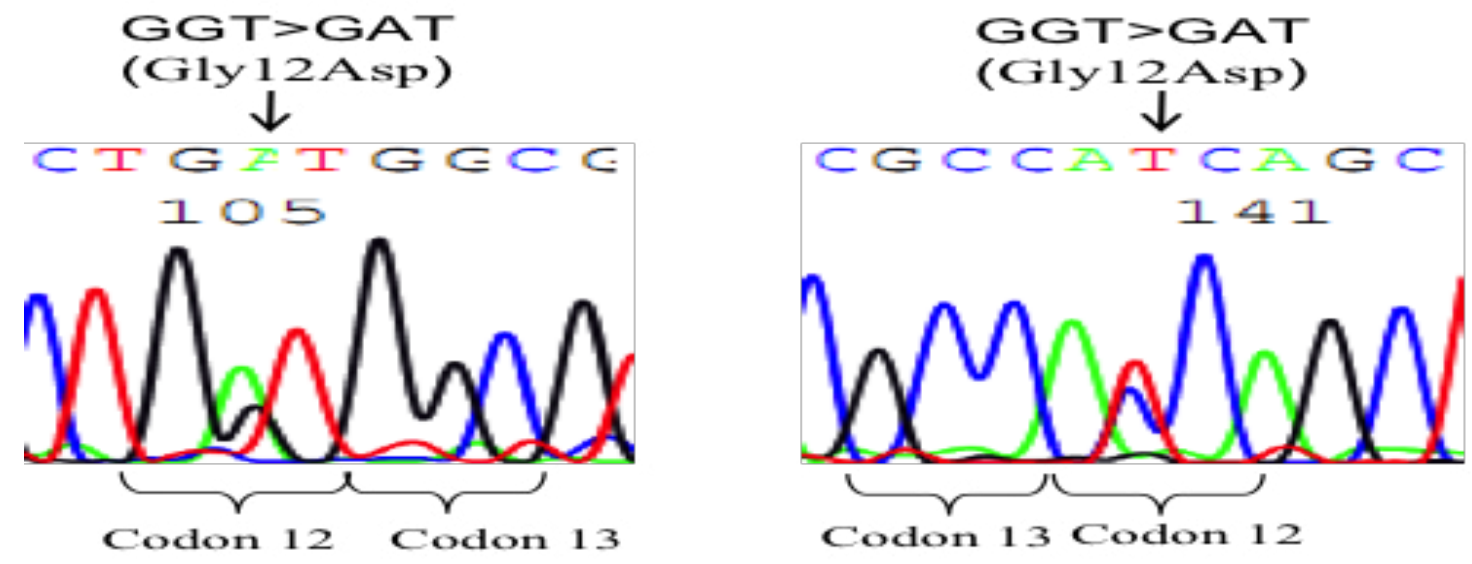


\section{DISSCUSION}

\section{Frequency of KRAS Mutations Among Patients with CRC}

The KRAS oncogene is mutated in approximately $35 \%-45 \%$ of colorectal cancers, and KRAS mutational status testing has been highlighted in recent years. The most frequent mutations in this gene, point substitutions in codons 12 and 13, were validated as negative predictors of response to anti-epidermal growth factor receptor antibodies. Therefore, determining the KRAS mutational status of tumor samples has become an essential tool for managing patients with colorectal cancers [5].

In our study population, the overall mutational rate for KRAS was $58.2 \%$, which seemed to be higher to those reported for other populations. Fu $\mathrm{X}$ et al [6], found KRAS exon 2 mutation rates were $37.7 \%$ in 5546 CRC patients diagnosed from 2010 to 2017. Guo $F$ et al [7], KRAS exon 2 mutations were identified in $42.2 \%$ in 353 CRC patients from two Chinese clinical centers. Hamzehzadeh L et [8], detected mutations in exon 2 (codons 12 and 13) of the KRAS and NRAS genes using high resolution melting analysis, Intplex design and Sanger sequencing in 87 Iranian CRC patients. Genomic DNA was isolated from fresh tissue samples of CRC patients. From 87 eligible cases, 51 were male and 36 were females. KRAS mutations in codons 12 and 13 were present in $28.7 \%$ of all analyzed CRCs. KRAS mutations were found in 37 (37.4\%) of 99 patients in the report of Inoue $Y$ et al [9]. The genetic analyses of KRAS gene found mutations in 22 cases (45.3\%) according to Dinu D et al [10].

As reported in 2011, the KRAS mutation frequencies in Asia, Europe, Latin American were 24\%, 36\% and $40 \%$, respectively $(P<0.0001)$ [11]. It is unclear why a lower incidence is observed in Asian patients.
In China, KRAS mutations were detected in 33.3\% (30/90) of the CRC tumor samples using the nucleotide sequence analysis method [12]. These results significantly correlated with the response rate and survival time of cetuximab-treated patients. The difference of mutation status may result from many aspects, such as the tissue, the percent of tumor cells, the extracted DNA quality, the testing methods and the testing target.

\section{Relationship Between KRAS Gene Mutation and Clinicopathologic Features}

The various rates of KRAS gene mutagenicity in CC that were detected in the tumor sub-localizations were $63.6 \%$ in the rectal (21 cases), $17.4 \%$ in the sigmoid colon (8 cases), $8.7 \%$ in the descending colon, transverse colon and/or hepatic flexure colon, $6.5 \%$ in the cecum (3 cases), and $2.2 \%$ in the lowest part of both the splenic flexure colon and ascending colon (1 case) ( $p>0.05$ ).

The rate of KRAS mutation in cancerous cells in the duodenum and colon were $45.7 \%$ and $54.3 \%$, respectively ( $p>0.05$ ). According to Breivik J. et al. (1994), 37.0\% of cancer patients expressed the KRAS mutation in the duodenum, while $41.3 \%$ expressed KRAS mutations in the colon ( $p>0.05$ ) [13]. Brink M. et al. (2003) determined the rate of KRAS mutational status in some regions of the colon, and showed that there percentages were $42 \%$ in the duodenum, $40 \%$ in the sigmoid colon, $17 \%$ in the proximal colon, and $38 \%$ in the distal colon $(P>0.05)$ [1]. However, Karapetis et al. (2008) showed that percentage of cancer patients expressing KRAS mutation only in the duodenum was $19.5 \%$, only in the colon was $65.9 \%$, and in both duodenum and colon was $14.6 \%$ [14].

Beranek M. et al. (1999) studied the KRAS mutations in the colon tumor sub-localizations and suggested that there was not any relation between KRAS

Table 2: The rate of KRAS gene mutation in some studies

\begin{tabular}{|l|c|c|}
\hline \multirow{2}{*}{ Authors } & \multicolumn{2}{|c|}{ Rate of KRAS Gene Mutation (\%) } \\
\cline { 2 - 3 } & Rectal \\
\hline Breivik J. et al (1994) (n=251) [13] & 37.0 & 41.3 \\
\hline Karapetis et al (2008) $(n=394)[14]$ & 19.5 & 65.9 \\
\hline Monstein và CS (2004) [16] & 30.0 & 44.0 \\
\hline Our data (2012), $(n=79)$ & 45.7 & 54.3 \\
\hline
\end{tabular}


gene mutation and sites in the colon $(p>0.05)$ [15]. Monstein et al. (2004) showed that the percentage of cancer patients with KRAS mutation in the duodenum was $30 \%$ and in the colon was $44 \%$ [16]. According to a study by Zulhabri O. et al. (2012), the rates of KRAS mutations in the colon tumor sub-localizations were $37 \%$ in the right colon and $14 \%$ in the left colon $(p>0.05)$ [17]. Hence, our results are consistent with these studies that found no correlation between the KRAS mutagenic rate in cancer patients and colon tumor sub-localization.

The rates of KRAS mutations in patients with tumor appearances were also evaluated. The mutations were found in $43.5 \%$ (20/46 cases) of ulcerated verrucous lesions, $26.1 \%$ (12/46 cases) of verrucous lesions, $13 \%$ (6/46 cases) of infiltrative lesions, and $8.7 \%$ (4/46 cases) of ulcerating lesions and infiltrative lesions. However, the differences in the rates were not significant $(p>0.05)$. The results of our study are consistent with the results of Beranek $M$. et al. (1999) in that the rate of KRAS mutation in cancer patients were not related to tumor forms [15]. Thus, there was no correlation between KRAS gene mutation with the tumor forms in the colon cancer patients.

Several studies have shown that the tumor size in combination with colon perimeter reflects tumor growth over time and is an independent prognostic factor for the patient's postoperative survival. The results showed that the rate of KRAS mutagenic mutation in CC patients with different tumor sizes are different. The rates of KRAS mutation were found in $63.1 \%$ ( 29 cases) of tumors with size $>10$ $\mathrm{cm}$, in $32.6 \%$ (15 cases) of tumors with size ranging from $5-10 \mathrm{~cm}$, and in $4.3 \%$ ( 2 cases) of tumors with size $<5 \mathrm{~cm}$. Thus, the rate of KRAS mutation in patients with CC patients gradually increased with tumor size; the differences were not statistically significant ( $p>0.05)$.

When studying the relationship between KRAS gene mutations in colon cancer patients with tumor invasion level (compared to the colon perimeter), the results showed that the rates of KRAS mutation in cancer patients were as follows: $63 \%$ (29 cases) in tumors accounting for $>3 / 4$ perimeter, $23.9 \%$ (11 cases) in tumors accounting for $1 / 2-3 / 4$ perimeter, $10.9 \%$ ( 5 cases) in tumors accounting for $1 / 4$ $1 / 2$ perimeter, $2.2 \%$ ( 1 case) of tumors accounting for $<1 / 4(p<0.05)$. Thus, the rate of KRAS mutation in cancer patients increased with tumor size.

Moreover, our results are consistent with the findings of Zulhabri O. et al. (2012) in the study of KRAS mutation at 12 codon sites in 70 colon cancer patients in Malaysia. Their study showed that the rate of KRAS gene mutations increased were associated with increasing tumor size. The percentage of KRAS mutation gradually increased from $10.0 \%$, to $17.0 \%$, and to $60.0 \%$, respectively, with the increase of tumor size from $<15$, to $15-34$, and to $>35 \mathrm{~cm} 2(p<0.05)$ [17]. Thus, there is a correlation between KRAS gene mutation and the size of the tumor in colon cancer patients.

The rate of KRAS mutation in colon cancer patients was also different in different types of tumors. For example, the rate of mutation was $43.3 \%$ in well-differentiated colorectal carcinoma, $32.6 \%$ in moderately differentiated colorectal carcinoma, $15.2 \%$ in poorly differentiated colorectal carcinoma, $8.7 \%$ in mucinous colorectal carcinoma, and $2.2 \%$ in signet ring adenocarcinoma. However, these differences were not statistiscally significant $(p>0.05)$.

The rate of KRAS mutation was also different among different malignancy grades but were also found to be non-significant $(p>0.05)$. The KRAS mutations were detected in $73.9 \%$ of patients with colon tumors of low malignancy grade and $26.1 \%$ in patients with colon tumors of high malignancy grade ( $p>0.05$ ). Thus, there was not any relation between KRAS mutation and histological types of tumors nor malignancy grades of the colon cancer.

We also evaluated the relation of KRAS mutation with the invasion levels of tumors into the bowel wall. The mutations were seen in $35.0 \%$ of cases of muscle layer invasion, $60.0 \%$ of cases of serosa invasion, and $70.6 \%$ of cases of subserosa invasion $(p<0.05)$. From these results, we can discern that there was a relation between KRAS gene mutation and tumor invasions in the colectoral carcinoma.

The relationship between the rate of patients with KRAS mutation and their status of lymph node metastasis were also investigated. The results as shown in Table 1 showed that there were $50.0 \%$ of colectoral carcinoma patients with lymph node metastasis exhibiting KRAS mutation, while there were $60.6 \%$ colectoral carcinoma patients with no lymph node metastasis exhibiting KRAS mutation ( $p>0.05$ ). Chang, M.Y. (2009) studied the KRAS mutation at codon 12 and 13 in colorectal cancer patients. Univariate analysis revealed a significant association between KRAS mutation at codon 12 and lymph node metastasis $(p=0.048)$. Multivariate analysis was adjusted for tumor size, histologic grade, and lymph node metastasis; the analysis showed that KRAS mutations at codon 12 and 13 correlate 
significantly with overall survival $(p=0.002)$ [18]. Thus the rate of KRAS mutations in colorectal cancer patients with lymph node metastasis and without lymph node metastasis are different among different studies. These results suggest that more studies with a greater number of patients are needed to better assess the association between KRAS mutation with status of lymph node metastasis.

\section{CONCLUSION}

The KRAS oncogene is mutated in $58.2 \%$ of colorectal cancers, and there was a relationship between KRAS mutational status and tumor size, tumor stage or metastatic stage. Therefore, determining the KRAS mutational status of tumor samples has become an essential tool for managing patients with colorectal cancers

\section{CONFLICT OF INTEREST STATEMENT}

The authors declare that they have no competing interests

\section{AUTHORS' CONTRIBUTION}

These authors who named below contributed equally to this work: Vu Thi Minh Thuc, Le Van Thieu, Huynh Quang Huy

[1] Brink M, de Goeij AF, Weijenberg MP, et al. K-ras oncogene mutations in sporadic colorectal cancer in The Netherlands Cohort Study. Carcinogenesis 2003; 24: 703-10.

[2] Anh PH. Cancer in Ha Noi. Vietnam Medical Journal 1993; 7: 14-21.

[3] Souglakos J. Genetic alterations in sporadic and hereditary colorectal cancer: implementations for screening and follow-up. Dig Dis 2007; 25: 9-19.

[4] Jackson EL, Willis N, Mercer K, et al. Analysis of lung tumor initiation and progression using conditional expression of oncogenic K-ras. Genes Dev 2001; 15: 3243-8.

[5] Tan C and Du X. KRAS mutation testing in metastatic colorectal cancer. World J Gastroenterol 2012; 18: 5171-80.

[6] Fu X, Huang Y, Fan X, et al. Demographic Trends and KRAS/ BRAF(V600E) Mutations in Colorectal Cancer Patients of South China: a Single-site Report. Int J Cancer 2019; 144: 2109-17.

[7] Guo F, Gong $\mathrm{H}$, Zhao H, et al. Mutation status and prognostic values of KRAS, NRAS, BRAF and PIK3CA in 353 Chinese colorectal cancer patients. Sci Rep 2018; 8: 6076.

[8] Hamzehzadeh L, Khadangi F, Ghayoor Karimiani E, et al. Common KRAS and NRAS gene mutations in sporadic colorectal cancer in Northeastern Iranian patients. Curr Probl Cancer 2018; 42: 572-81.

[9] Inoue $Y$, Saigusa $S$, Iwata $T$, et al. The prognostic value of KRAS mutations in patients with colorectal cancer. Oncol Rep 2012; 28: 1579-84.

[10] Dinu D, Dobre M, Panaitescu E, et al. Prognostic significance of
KRAS gene mutations in colorectal cancer--preliminary study. J Med Life 2014; 7: 581-87.

[11] Ciardiello F, Tejpar S, Normanno N, et al. Uptake of KRAS mutation testing in patients with metastatic colorectal cancer in Europe, Latin America and Asia. Target Oncol 2011; 6: 133-45.

[12] Li FH, Shen L, Li ZH, et al. Impact of KRAS mutation and PTEN expression on cetuximab-treated colorectal cancer. World J Gastroenterol 2010; 16: 5881-88.

[13] Breivik J, Meling Gl, Spurkland A, et al. K-ras mutation in colorectal cancer: relations to patient age, sex and tumour location. $\mathrm{Br} J$ Cancer 1994; 69: 367-71.

[14] Karapetis CS, Khambata-Ford S, Jonker DJ, et al. K-ras mutations and benefit from cetuximab in advanced colorectal cancer. $\mathrm{N}$ Engl J Med 2008; 359: 1757-65.

[15] Beranek M, Bures J, Palicka V, et al. A relationship between K-ras gene mutations and some clinical and histologic variables in patients with primary colorectal carcinoma. Clin Chem Lab Med 1999; 37: 723-27.

[16] Monstein HJ, Fransen K, Dimberg J, et al. K-ras and B-raf gene mutations are not associated with gastrin- and CCK2-receptor mRNA expression in human colorectal tumour tissues. Eur J Clin Invest 2004; 34: 100-06.

[17] Zulhabri O, Rahman J, Ismail S, et al. Predominance of $G$ to $A$ codon 12 mutation K-ras gene in Dukes' B colorectal cancer. Singapore Med J 2012; 53: 26-31.

[18] [Chang YS, Yeh KT, Chang TJ, et al. Fast simultaneous detection of K-RAS mutations in colorectal cancer. BMC Cancer 2009; 9: 179 